GENETIC SCREENS

\section{Finding the resilient few}

Researchers of a study published in Nature Biotechnology have identified 13 healthy individuals who harbour genetic variants associated with severe Mendelian diseases that were previously thought to be completely penetrant.

Studies of disease genetics are generally focused on the identification of disease-causing variants in affected individuals. Chen et al. took a different approach, and instead looked for disease mutations in healthy individuals, with a view to identifying factors that suppress the effects of disease-causing mutations.

The group analysed whole-genome sequencing, whole-exome sequencing and array-based genotyping data sets from 12 studies encompassing 589,306 healthy individuals. To minimize the chance of false positives due to missed diagnoses, they focused on mutations annotated as completely penetrant for severe childhood diseases and only included data from adults. The data sets were screened using a gene panel comprising 784 genes implicated in 584 Mendelian diseases falling into 17 different disease categories.

Screening all 589,306 sets of genomic data identified 15,597 samples as belonging to candidate resilient individuals. The team excluded 15,294 of these samples owing to low confidence in the variant call, because the variant identified was present at high frequency in the population or because data were not available for follow up.

The remaining 303 candidates were reviewed individually by a team comprising bioinformaticians, clinical geneticists, consultants and genetic counsellors, to determine whether these candidates could be classified as completely resilient. 261 candidates were excluded because of insufficient evidence in the literature of a pathogenic phenotype for the disease variant identified or because the associated disease phenotype was insufficiently severe. A further 29 candidates were excluded based on evidence of the expected disease phenotypes in their medical records or because Sanger sequencing revealed that the sample had been incorrectly genotyped as homozygous based on the original variant data.

A final 13 candidate resilient individuals each had disease mutations that are implicated in 1 out of 8 different Mendelian diseases that would be expected to manifest severe phenotypes in childhood, such as cystic fibrosis. It seems highly unlikely that such extreme disease manifestations would be omitted from medical records, suggesting that these individuals are truly resistant to the effects of severe disease-causing mutations that were believed to be completely penetrant, and that incomplete penetrance is more common than previously thought.

A major stumbling block in this study was the inability to recontact the candidate resilient individuals in order to further confirm their status, owing to a lack of the required clause in the consent forms used in most of the original studies. The team were therefore unable to rule out alternative explanations for the observed discrepancies between genotype and phenotype, such as somatic mosaicism, delayed diagnosis or mismatched DNA samples and records.

Nonetheless, this study highlights the potential of broader studies to systematically screen for healthy individuals who are resistant to highly penetrant disease-causing mutations, as well as the importance of seeking appropriate consent for further contact. The study of resilient individuals could enable the elucidation of Mendelian disease mechanisms and the identification of second-site mutations that buffer the effects of deleterious variants, and thus have potential as therapeutic targets.

Denise Waldron

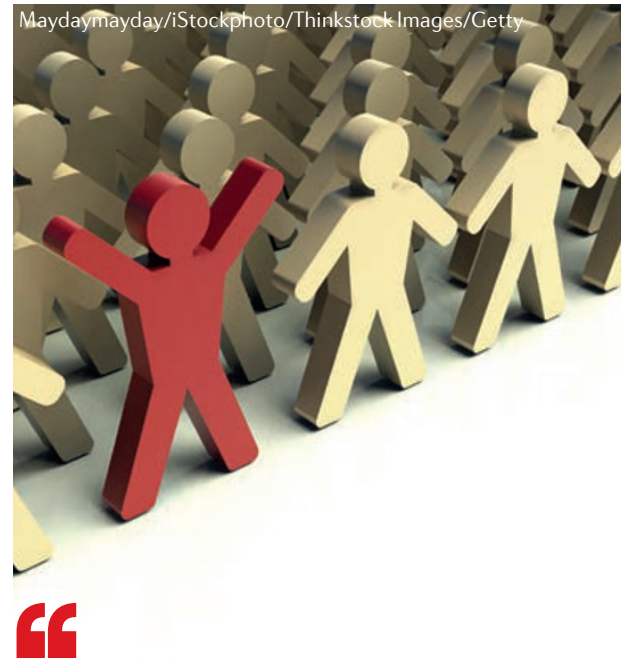

13 candidate resilient individuals each had disease mutations that are implicated in 1 out of 8 different Mendelian diseases that would be expected to manifest severe phenotypes in childhood 